\title{
Search for single top quark production via contact interactions at LEP2
}

\author{
The DELPHI Collaboration
}

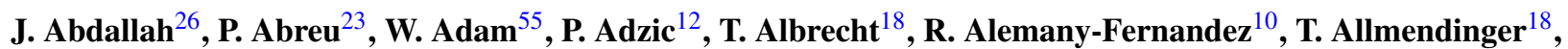
P.P. Allport ${ }^{24}$, U. Amaldi ${ }^{30}$, N. Amapane ${ }^{48}$, S. Amato ${ }^{52}$, E. Anashkin ${ }^{37}$, A. Andreazza ${ }^{29}$, S. Andringa ${ }^{23}$, N. Anjos ${ }^{23}$, P. Antilogus ${ }^{26}$, W-D. Apel ${ }^{18}$, Y. Arnoud ${ }^{15}$, S. Ask ${ }^{10}$, B. Asman ${ }^{47}$, J.E. Augustin ${ }^{26}$, A. Augustinus ${ }^{10}$, P. Baillon ${ }^{10}$, A. Ballestrero ${ }^{49}$, P. Bambade ${ }^{21}$, R. Barbier ${ }^{28}$, D. Bardin ${ }^{17}$, G.J. Barker ${ }^{\mathrm{d}}$, A. Baroncelli ${ }^{40}$, M. Battaglia ${ }^{10}$, M. Baubillier ${ }^{26}$, K-H. Becks ${ }^{57}$, M. Begalli ${ }^{8}$, A. Behrmann ${ }^{57}$, E. Ben-Haim ${ }^{26}$, N. Benekos ${ }^{33}$, A. Benvenuti ${ }^{6}$, C. Berat ${ }^{15}$, M. Berggren ${ }^{26}$, D. Bertrand ${ }^{3}$, M. Besancon ${ }^{41}$, N. Besson ${ }^{41}$, D. Bloch ${ }^{11}$, M. Blom ${ }^{32}$, M. Bluj $^{56}$, M. Bonesini ${ }^{30}$, M. Boonekamp ${ }^{41}$, P.S.L. Booth ${ }^{24, b}$, G. Borisov ${ }^{22}$, O. Botner $^{53}$, B. Bouquet ${ }^{21}$, T.J.V. Bowcock ${ }^{24}$, I. Boyko $^{17}$, M. Bracko ${ }^{44}$, R. Brenner ${ }^{53}$, E. Brodet ${ }^{36}$, P. Bruckman ${ }^{19}$, J.M. Brunet ${ }^{9}$, B. Buschbeck ${ }^{55}$, P. Buschmann ${ }^{57}$, M. Calvi ${ }^{30}$, T. Camporesi $^{10}$, V. Canale ${ }^{39}$, F. Carena ${ }^{10}$, N. Castro ${ }^{23}$, F. Cavallo ${ }^{6}$, M. Chapkin ${ }^{43}$, Ph. Charpentier ${ }^{10}$, P. Checchia ${ }^{37}$, R. Chierici $^{10}$, P. Chliapnikov ${ }^{43}$, J. Chudoba ${ }^{10}$, S.U. Chung ${ }^{10}$, K. Cieslik ${ }^{19}$, P. Collins ${ }^{10}$, R. Contri ${ }^{14}$, G. Cosme ${ }^{21}$, F. Cossutti ${ }^{50}$, M.J. Costa ${ }^{54}$, D. Crennell ${ }^{38}$, J. Cuevas ${ }^{35}$, J. D'Hondt ${ }^{3}$, T. da Silva ${ }^{52}$, W. Da Silva ${ }^{26}$, G. Della Ricca ${ }^{50}$, A. De Angelis ${ }^{51}$, W. De Boer ${ }^{18}$, C. De Clercq ${ }^{3}$, B. De Lotto ${ }^{51}$, N. De Maria ${ }^{48}$, A. De Min ${ }^{37}$, L. de Paula ${ }^{52}$, L. Di Ciaccio ${ }^{39}$, A. Di Simone ${ }^{40}$, K. Doroba ${ }^{56}$, J. Drees ${ }^{57}$, G. Eigen ${ }^{5}$, T. Ekelof ${ }^{53}$, M. Ellert $^{53}$, M. Elsing ${ }^{10}$, M.C. Espirito Santo ${ }^{23}$, G. Fanourakis ${ }^{12}$, D. Fassouliotis ${ }^{12,4}$, M. Feindt ${ }^{18}$, J. Fernandez Fe A. Ferrer $^{54}$, F. Ferro ${ }^{14}$, U. Flagmeyer ${ }^{57}$, H. Foeth ${ }^{10}$, E. Fokitis ${ }^{33}$, F. Fulda-Quenzer ${ }^{21}$, J. Fuster ${ }^{54}$, M. Gandelman ${ }^{52}$, C. Garcia $^{54}$, Ph. Gavillet ${ }^{10}$, E. Gazis ${ }^{33}$, R. Gokieli ${ }^{10,56}$, B. Golob ${ }^{44,46}$, G. Gomez-Ceballos ${ }^{42}$, P. Goncalves ${ }^{23}$, E. Graziani $^{40}$, G. Grosdidier ${ }^{21}$, K. Grzelak ${ }^{56}$, J. Guy ${ }^{38}$, C. Haag ${ }^{18}$, A. Hallgren ${ }^{53}$, K. Hamacher ${ }^{57}$, K. Hamilton ${ }^{36}$, S. Haug ${ }^{34}$, F. Hauler ${ }^{18}$, V. Hedberg ${ }^{27}$, M. Hennecke ${ }^{18}$, J. Hoffman ${ }^{56}$, S-O. Holmgren ${ }^{47}$, P.J. Holt ${ }^{10}$, M.A. Houlden ${ }^{24}$, J.N. Jackson ${ }^{24}$, G. Jarlskog ${ }^{27}$, P. Jarry ${ }^{41}$, D. Jeans ${ }^{36}$, E.K. Johansson ${ }^{47}$, P. Jonsson ${ }^{28}$, C. Joram ${ }^{10}$, L. Jungermann ${ }^{18}$, F. Kapusta ${ }^{26}$, S. Katsanevas ${ }^{28}$, E. Katsoufis ${ }^{33}$, G. Kernel ${ }^{44}$, B.P. Kersevan ${ }^{44,46}$, U. Kerzel ${ }^{18}$, B.T. King ${ }^{24}$, N.J. Kjaer ${ }^{10}$, P. Kluit ${ }^{32}$, P. Kokkinias ${ }^{12}$, C. Kourkoumelis ${ }^{4}$, O. Kouznetsov ${ }^{17}$, Z. Krumstein ${ }^{17}$, M. Kucharczyk ${ }^{19}$, J. Lamsa ${ }^{1}$, G. Leder ${ }^{55}$, F. Ledroit ${ }^{15}$, L. Leinonen ${ }^{47}$, R. Leitner ${ }^{31}$, J. Lemonne ${ }^{3}$, V. Lepeltier ${ }^{21, b}$, T. Lesiak ${ }^{19}$, W. Liebig ${ }^{57}$, D. Liko ${ }^{55}$, A. Lipniacka ${ }^{47}$, J.H. Lopes ${ }^{52}$, J.M. Lopez ${ }^{35}$, D. Loukas ${ }^{12}$, P. Lutz ${ }^{41}$, L. Lyons ${ }^{36}$, J. MacNaughton ${ }^{55}$,

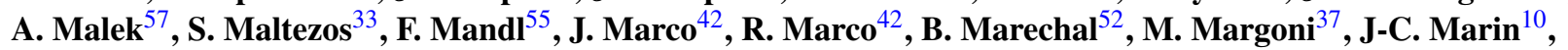
C. Mariotti $^{10}$, A. Markou ${ }^{12}$, C. Martinez-Rivero ${ }^{42}$, J. Masik ${ }^{13}$, N. Mastroyiannopoulos ${ }^{12}$, F. Matorras $^{42}$, C. Matteuzzi $^{30}$, F. Mazzucato ${ }^{37}$, M. Mazzucato ${ }^{37}$, R. Mc Nulty ${ }^{24}$, C. Meroni ${ }^{29}$, E. Migliore ${ }^{48}$, W. Mitaroff ${ }^{55}$,

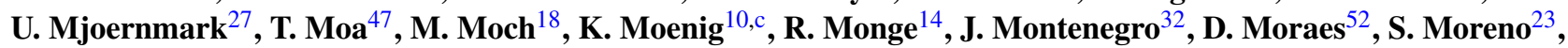
P. Morettini $^{14}$, U. Mueller ${ }^{57}$, K. Muenich ${ }^{57}$, M. Mulders ${ }^{32}$, L. Mundim ${ }^{8}$, W. Murray ${ }^{38}$, B. Muryn ${ }^{20}$, G. Myatt ${ }^{36}$, T. Myklebust ${ }^{34}$, M. Nassiakou ${ }^{12}$, F. Navarria ${ }^{6}$, K. Nawrocki ${ }^{56}$, S. Nemecek ${ }^{13}$, R. Nicolaidou ${ }^{41}$, M. Nikolenko $^{17,11}$, A. Oblakowska-Mucha ${ }^{20}$, V. Obraztsov ${ }^{43}$, O. Oliveira ${ }^{23}$, A. Olshevski ${ }^{17}$, A. Onofre ${ }^{23}$, R. Orava ${ }^{16}$ K. Osterberg $^{16}$, A. Ouraou ${ }^{41}$, A. Oyanguren ${ }^{54}$, M. Paganoni ${ }^{30}$, S. Paiano ${ }^{6}$, J.P. Palacios ${ }^{24}$, H. Palka ${ }^{19}$, Th.D. Papadopoulou ${ }^{33}$, L. Pape $^{10}$, C. Parkes $^{25}$, F. Parodi ${ }^{14}$, U. Parzefall ${ }^{10}$, A. Passeri ${ }^{40}$, O. Passon ${ }^{57}$, L. Peralta ${ }^{23}$, V. Perepelitsa ${ }^{54}$, A. Perrotta $^{6}$, A. Petrolini ${ }^{14}$, J. Piedra ${ }^{42}$, L. Pieri ${ }^{40}$, F. Pierre ${ }^{41, b}$, M. Pimenta ${ }^{23}$, E. Piotto ${ }^{10}$, T. Podobnik $^{44,46}$, V. Poireau ${ }^{10}$, M.E. Pol ${ }^{7}$, G. Polok ${ }^{19}$, V. Pozdniakov ${ }^{17}$, N. Pukhaeva ${ }^{17}$, A. Pullia ${ }^{30}$, D. Radojicic ${ }^{36}$, P. Rebecchi $^{10}$, J. $\operatorname{Rehn}^{18}$, D. Reid $^{32}$, R. Reinhardt ${ }^{57}$, P. $\operatorname{Renton}^{36}$, F. Richard ${ }^{21}$, J. Ridky ${ }^{13}$, M. Rivero ${ }^{42}$, D. Rodriguez $^{42}$, A. Romero $^{48}$, P. Ronchese $^{37}$, P. Roudeau ${ }^{21}$, T. Rovelli ${ }^{6}$, V. Ruhlmann-Kleider ${ }^{41}$, D. Ryabtchikov ${ }^{43}$, A. Sadovsky $^{17}$, L. Salmi ${ }^{16}$, J. Salt ${ }^{54}$, C. Sander ${ }^{18}$, A. Savoy-Navarro ${ }^{26}$, U. Schwickerath ${ }^{10}$, R. Sekulin ${ }^{38}$, M. Siebel ${ }^{57}$, A. Sisakian ${ }^{17}$, G. Smadja ${ }^{28}$, O.Smirnova ${ }^{27}$, A. Sokolov ${ }^{43}$, A. Sopczak ${ }^{22}$, R. Sosnowski ${ }^{56}$, T. Spassov ${ }^{10}$, M. Stanitzki ${ }^{18}$, A. Stocchi ${ }^{21}$, J. Strauss ${ }^{55}$, B. Stugu ${ }^{5}$, M. Szczekowski ${ }^{56}$, M. Szeptycka ${ }^{56}$, T. Szumlak $^{20}$, T. Tabarelli ${ }^{30}$, F. Tegenfeldt $^{53}$,

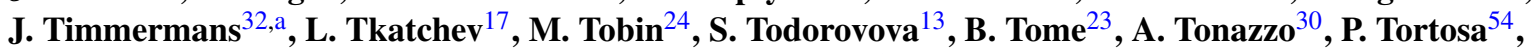
P. Travnicek ${ }^{13}$, D. Treille ${ }^{10}$, G. Tristram ${ }^{9}$, M. Trochimczuk ${ }^{56}$, C. Troncon $^{29}$, M-L. Turluer ${ }^{41}$, I.A. Tyapkin ${ }^{17}$, P. Tyapkin ${ }^{17}$, S. Tzamarias ${ }^{12}$, V. Uvarov ${ }^{43}$, G. Valenti ${ }^{6}$, P. Van Dam ${ }^{32}$, J. Van Eldik ${ }^{10}$, N. van Remortel ${ }^{2}$,

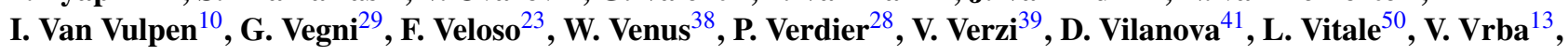


H. Wahlen ${ }^{57}$, A.J. Washbrook ${ }^{24}$, C. Weiser ${ }^{18}$, D. Wicke ${ }^{10}$, J. Wickens ${ }^{3}$, G. Wilkinson ${ }^{36}$, M. Winter ${ }^{11}$, M. Witek ${ }^{19}$, O. Yushchenko ${ }^{43}$, A. Zalewska ${ }^{19}$, P. Zalewski ${ }^{56}$, D. Zavrtanik ${ }^{45}$, V. Zhuravlov ${ }^{17}$, N.I. Zimin ${ }^{17}$, A. Zintchenko ${ }^{17}$, M. Zupan ${ }^{12}$

${ }^{1}$ Department of Physics and Astronomy, Iowa State University, Ames, IA 50011-3160, USA

${ }^{2}$ Physics Department, Universiteit Antwerpen, Universiteitsplein 1, 2610 Antwerpen, Belgium

${ }^{3}$ IIHE, ULB-VUB, Pleinlaan 2, 1050 Brussels, Belgium

${ }^{4}$ Physics Laboratory, University of Athens, Solonos Str. 104, 10680 Athens, Greece

${ }^{5}$ Department of Physics, University of Bergen, Allégaten 55, 5007 Bergen, Norway

${ }^{6}$ Dipartimento di Fisica, Università di Bologna and INFN, Viale C. Berti Pichat 6/2, 40127 Bologna, Italy

${ }^{7}$ Centro Brasileiro de Pesquisas Físicas, rua Xavier Sigaud 150, 22290 Rio de Janeiro, Brazil

${ }^{8}$ Inst. de Física, Univ. Estadual do Rio de Janeiro, rua São Francisco Xavier 524, Rio de Janeiro, Brazil

${ }^{9}$ Collège de France, Lab. de Physique Corpusculaire, IN2P3-CNRS, 75231 Paris Cedex 05, France

${ }^{10}$ CERN, 1211 Geneva 23, Switzerland

${ }^{11}$ Institut Pluridisciplinaire Hubert Curien, Université de Strasbourg, IN2P3-CNRS, BP28, 67037 Strasbourg Cedex 2, France

${ }^{12}$ Institute of Nuclear Physics, N.C.S.R. Demokritos, P.O. Box 60228, 15310 Athens, Greece

${ }^{13}$ FZU, Inst. of Phys. of the C.A.S. High Energy Physics Division, Na Slovance 2, 182 21, Praha 8, Czech Republic

${ }_{15}^{14}$ Dipartimento di Fisica, Università di Genova and INFN, Via Dodecaneso 33, 16146 Genova, Italy

${ }^{15}$ Institut des Sciences Nucléaires, IN2P3-CNRS, Université de Grenoble 1, 38026 Grenoble Cedex, France

${ }^{16}$ Helsinki Institute of Physics and Department of Physical Sciences, P.O. Box 64, 00014 University of Helsinki, Finland

${ }^{17}$ Joint Institute for Nuclear Research, Dubna, Head Post Office, P.O. Box 79, 101000 Moscow, Russian Federation

${ }^{18}$ Institut für Experimentelle Kernphysik, Universität Karlsruhe, Postfach 6980, 76128 Karlsruhe, Germany

${ }^{19}$ Institute of Nuclear Physics PAN, Ul. Radzikowskiego 152, 31142 Krakow, Poland

${ }^{20}$ Faculty of Physics and Nuclear Techniques, University of Mining and Metallurgy, 30055 Krakow, Poland

${ }^{21}$ LAL, Univ. Paris-Sud, CNRS/IN2P3, Orsay, France

${ }^{22}$ School of Physics and Chemistry, University of Lancaster, Lancaster LA1 4YB, UK

${ }^{23}$ LIP, FCUL, IST, CFC-UC, Av. Elias Garcia, 14-1 ${ }^{\circ}, 1000$ Lisboa Codex, Portugal

${ }^{24}$ Department of Physics, University of Liverpool, P.O. Box 147, Liverpool L69 3BX, UK

${ }_{25}^{25}$ Department of Physics and Astronomy, Kelvin Building, University of Glasgow, Glasgow G12 8QQ, UK

${ }^{26}$ LPNHE, IN2P3-CNRS, Univ. Paris VI et VII, Tour 33 (RdC), 4 place Jussieu, 75252 Paris Cedex 05, France

${ }^{27}$ Department of Physics, University of Lund, Sölvegatan 14, 22363 Lund, Sweden

${ }^{28}$ Université Claude Bernard de Lyon, IPNL, IN2P3-CNRS, 69622 Villeurbanne Cedex, France

${ }^{29}$ Dipartimento di Fisica, Università di Milano and INFN-MILANO, Via Celoria 16, 20133 Milan, Italy

${ }^{30}$ Dipartimento di Fisica, Università di Milano-Bicocca and INFN-MILANO, Piazza della Scienza 3, 20126 Milan, Italy

${ }^{31}$ IPNP of MFF, Charles University, Areal MFF, V Holesovickach 2, 180 00, Praha 8, Czech Republic

${ }^{32}$ NIKHEF, Postbus 41882, 1009 DB Amsterdam, The Netherlands

${ }_{33}^{33}$ National Technical University, Physics Department, Zografou Campus, 15773 Athens, Greece

${ }^{34}$ Physics Department, University of Oslo, Blindern, 0316 Oslo, Norway

${ }^{35}$ Departamento de la Fisica, Universidad Oviedo, Avda. Calvo Sotelo s/n, 33007 Oviedo, Spain

${ }^{36}$ Department of Physics, University of Oxford, Keble Road, Oxford OX1 3RH, UK

${ }^{37}$ Dipartimento di Fisica, Università di Padova and INFN, Via Marzolo 8, 35131 Padua, Italy

${ }^{38}$ Rutherford Appleton Laboratory, Chilton, Didcot OX11 OQX, UK

${ }^{39}$ Dipartimento di Fisica, Università di Roma II and INFN, Tor Vergata, 00173 Rome, Italy

${ }^{40}$ Dipartimento di Fisica, Università di Roma III and INFN, Via della Vasca Navale 84, 00146 Rome, Italy

${ }^{41}$ DAPNIA/Service de Physique des Particules, CEA-Saclay, 91191 Gif-sur-Yvette Cedex, France

${ }^{42}$ Instituto de Fisica de Cantabria (CSIC-UC), Avda. los Castros s/n, 39006 Santander, Spain

${ }^{43}$ Institute for High Energy Physics, Serpukov P.O. Box 35, Protvino, Moscow Region, Russian Federation

${ }^{44}$ J. Stefan Institute, Jamova 39, 1000 Ljubljana, Slovenia

${ }^{45}$ Laboratory for Astroparticle Physics, University of Nova Gorica, Kostanjeviska 16a, 5000 Nova Gorica, Slovenia

${ }^{46}$ Department of Physics, University of Ljubljana, 1000 Ljubljana, Slovenia

${ }^{47}$ Fysikum, Stockholm University, Box 6730, 11385 Stockholm, Sweden

${ }^{48}$ Dipartimento di Fisica Sperimentale, Università di Torino and INFN, Via P. Giuria 1, 10125 Turin, Italy

${ }^{49}$ INFN, Sezione di Torino and Dipartimento di Fisica Teorica, Università di Torino, Via Giuria 1, 10125 Turin, Italy

${ }^{50}$ Dipartimento di Fisica, Università di Trieste and INFN, Via A. Valerio 2, 34127 Trieste, Italy

${ }^{51}$ Istituto di Fisica, Università di Udine and INFN, 33100 Udine, Italy

${ }^{52}$ Univ. Federal do Rio de Janeiro, C.P. 68528 Cidade Univ., Ilha do Fundão 21945-970 Rio de Janeiro, Brazil

${ }_{54}^{53}$ Department of Radiation Sciences, University of Uppsala, P.O. Box 535, 75121 Uppsala, Sweden

${ }_{55}^{54}$ IFIC, Valencia-CSIC, and D.F.A.M.N., U. de Valencia, Avda. Dr. Moliner 50, 46100 Burjassot (Valencia), Spain

${ }^{55}$ Institut für Hochenergiephysik, Österr. Akad. d. Wissensch., Nikolsdorfergasse 18, 1050 Vienna, Austria

${ }^{56}$ Institute Nuclear Studies and University of Warsaw, U1. Hoza 69, 00681 Warsaw, Poland

${ }^{57}$ Fachbereich Physik, University of Wuppertal, Postfach 100 127, 42097 Wuppertal, Germany 
Abstract Single top quark production via four-fermion contact interactions associated to flavour-changing neutral currents was searched for in data taken by the DELPHI detector at LEP2. The data were accumulated at centre-ofmass energies ranging from 189 to $209 \mathrm{GeV}$, with an integrated luminosity of $598.1 \mathrm{pb}^{-1}$. No evidence for a signal was found. Limits on the energy scale $\Lambda$, were set for scalar-, vector- and tensor-like coupling scenarios.

\section{Introduction}

With a mass of $173.3 \pm 0.6 \pm 0.9 \mathrm{GeV}$ [1], the $\mathrm{t}$ quark is the heaviest known one and, due to its large mass, the most sensitive to new physics. In $\mathrm{e}^{+} \mathrm{e}^{-}$collisions at LEP2, $\mathrm{t}$ quarks could only be singly produced, due to the limited centreof-mass energy. In the Standard Model (SM) they would be generated in association with $\mathrm{b}$ or $\mathrm{c}$ quarks, through the processes ${ }^{1} \mathrm{e}^{+} \mathrm{e}^{-} \rightarrow \mathrm{tb}^{-} \bar{\nu}_{\mathrm{e}}$ and $\mathrm{e}^{+} \mathrm{e}^{-} \rightarrow \mathrm{t} \overline{\mathrm{c}}$. A complete tree level calculation has shown that the cross-section of the first process is at the level of $10^{-6} \mathrm{pb}$ [2]. The second process proceeds via Flavour Changing Neutral Currents (FCNC), which are forbidden at tree level and suppressed by the GIM mechanism [3] at higher orders. The corresponding crosssection is of the order of $10^{-12} \mathrm{pb}$ [4].

Enhanced $\mathrm{e}^{+} \mathrm{e}^{-} \rightarrow \mathrm{t} \overline{\mathrm{c}}$ cross-sections (or top FCNC branching ratios) are, however, foreseen in several new physics scenarios, such as models with extra $Q=2 / 3$ quark singlets [5], two Higgs doublet models (2HDM) [6, 7], flavour conserving $2 \mathrm{HDM}[7,8]$, minimal supersymmetric SM [9-11] or non-minimal supersymmetric models with $R$ parity violation [12]. Single t quark production at LEP2 would thus be a signature of new physics.

The four LEP collaborations [13-17] searched previously for single t production in the context of Ref. [18]. In the model used, single $t$ production is described in terms of vector-like anomalous couplings ( $\kappa_{\mathrm{Z}}$ and $\kappa_{\gamma}$ ) associated with the already known $\mathrm{Z}$ and $\gamma$ bosons. The physics energy scale was set to the $t$ mass.

In this paper, a very general approach describing single $\mathrm{t}$ quark production via $\mathrm{e}^{+} \mathrm{e}^{-} \rightarrow \mathrm{t} \overline{\mathrm{c}}$ through an effective Lagrangian with FCNC operators is used [19]. Apart from the $\mathrm{Z}$ and $\gamma$ bosons, new four-fermion contact interactions, which include additional scalar-, vector-, and tensorlike couplings, are possible. The contribution of the $\mathrm{Z}$ boson

\footnotetext{
${ }^{1}$ Throughout this paper the charge conjugated processes are also included, unless explicitly stated otherwise.

a e-mail: timmerma@mail.cern.ch

${ }^{\mathrm{b}}$ Deceased.

${ }^{\mathrm{c}}$ Now at DESY-Zeuthen, Platanenallee 6, 15735 Zeuthen, Germany.

${ }^{\mathrm{d}}$ Now at University of Warwick, Coventry CV4 7AL, UK.
}

is also included, through a vector-like coupling which can be related to the anomalous coupling $\kappa_{\mathrm{Z}}$ [18]. The physics energy scale is a free parameter in this model. The kinematic differences between different coupling assumptions are taken into account and a dedicated analysis is developed.

This paper is organized as follows: single t quark production and decay is briefly discussed in Sect. 2. In Sect. 3 the data sets and the simulated samples are presented. The analysis is described in Sect. 4 and the results are presented in Sect. 5. In Sect. 6, conclusions are drawn and the results are compared with previous LEP studies.

\section{Single t quark production and decay}

The process $\mathrm{e}^{+} \mathrm{e}^{-} \rightarrow \mathrm{t} \overline{\mathrm{c}}$ can be described by an effective Lagrangian with FCNC operators [19]. Figure 1 shows the Feynman diagrams considered in this model. New contact interaction terms are associated to new scalar $\left(S_{R R}\right)$, vector $\left(V_{i j}, i, j=L, R\right)$ and tensor-like $\left(T_{R R}\right)$ couplings of heavy fields, and a term describing a new Ztc vertex parametrized by vector $\left(a_{j}^{Z}\right)$ couplings is introduced.

The differential cross-section, for tic production only, can be obtained from the Lagrangian given in Ref. [19] and is expressed in terms of the couplings and of a new physics energy scale parameter $\Lambda$ :

$$
\begin{aligned}
\frac{\mathrm{d} \sigma}{\mathrm{d} \cos \theta} & \left(\mathrm{e}^{+} \mathrm{e}^{-} \rightarrow \mathrm{t} \overline{\mathrm{c}}\right) \\
= & \frac{3 \mathcal{C}}{8}\left\{S_{R R}^{2}(1+\beta)-4 S_{R R} T_{R R}(1+\beta) \cos \theta\right. \\
& +16 T_{R R}^{2}\left(1-\beta+2 \beta \cos ^{2} \theta\right) \\
& +2\left[\left(V_{R R}+4 c_{R}^{Z} a_{R}^{Z} \frac{m_{W} m_{Z}}{s-m_{Z}^{2}}\right)^{2}\right. \\
& \left.+\left(V_{L L}+4 c_{L}^{Z} a_{L}^{Z} \frac{m_{W} m_{Z}}{s-m_{Z}^{2}}\right)^{2}\right] \\
& \times\left[1+(1+\beta) \cos \theta+\beta \cos ^{2} \theta\right] \\
& +2\left[\left(V_{R L}+4 c_{R}^{Z} a_{L}^{Z} \frac{m_{W} m_{Z}}{s-m_{Z}^{2}}\right)^{2}\right. \\
& \left.+\left(V_{L R}+4 c_{L}^{Z} a_{R}^{Z} \frac{m_{W} m_{Z}}{s-m_{Z}^{2}}\right)^{2}\right] \\
& \left.\times\left[1-(1+\beta) \cos \theta+\beta \cos ^{2} \theta\right]\right\}
\end{aligned}
$$

where

$$
\begin{gathered}
\mathcal{C}=\frac{s}{\Lambda^{4}} \frac{\beta^{2}}{4 \pi(1+\beta)^{3}}, \quad \beta=\frac{\left(s-m_{\mathrm{t}}^{2}\right)}{\left(s+m_{\mathrm{t}}^{2}\right)}, \\
c_{L}^{Z}=-1 / 2+\sin ^{2} \theta_{W}, \quad c_{R}^{Z}=\sin ^{2} \theta_{W},
\end{gathered}
$$




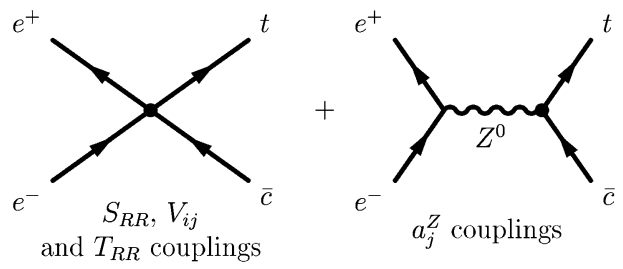

Fig. 1 The eetc and Ztc vertex contributions to the $\mathrm{e}^{+} \mathrm{e}^{-} \rightarrow$ tc process

$\beta$ is the velocity of the outgoing t quark, $\theta_{\mathrm{W}}$ is the electroweak mixing angle and $\theta$ is the angle between the incident electron beam and the $t$ quark. The coupling scenarios listed in Table 1 were considered in this study. The " $V+a$ " (" $V-a$ ") scenario corresponds to the constructive (destructive) interference between the eetc and the Ztc vertices. The differential cross-section depends on the coupling scenarios as can be seen in Fig. 2 for scenarios $S V T, S, V$ and $T$, considering $m_{\mathrm{t}}=175 \mathrm{GeV} / c^{2}, \Lambda=1 \mathrm{TeV}$ and $\sqrt{s}=206 \mathrm{GeV}$.

The total production cross-section, including charge conjugation, obtained from (1) is

$$
\begin{aligned}
& \sigma\left(\mathrm{e}^{+} \mathrm{e}^{-} \rightarrow \mathrm{t} \mathrm{c}\right)+\sigma\left(\mathrm{e}^{+} \mathrm{e}^{-} \rightarrow \overline{\mathrm{t}}\right) \\
&=\mathcal{C}\left\{8(3-\beta) T_{R R}^{2}+\frac{3}{2}(1+\beta) S_{R R}^{2}+(3+\beta)\right. \\
& \times\left[\left(V_{R R}+4 c_{R}^{Z} a_{R}^{Z} \frac{m_{W} m_{Z}}{s-m_{Z}^{2}}\right)^{2}\right. \\
&+\left(V_{L L}+4 c_{L}^{Z} a_{L}^{Z} \frac{m_{W} m_{Z}}{s-m_{Z}^{2}}\right)^{2} \\
&+\left(V_{R L}+4 c_{R}^{Z} a_{L}^{Z} \frac{m_{W} m_{Z}}{s-m_{Z}^{2}}\right)^{2} \\
&\left.\left.+\left(V_{L R}+4 c_{L}^{Z} a_{R}^{Z} \frac{m_{W} m_{Z}}{s-m_{Z}^{2}}\right)^{2}\right]\right\} .
\end{aligned}
$$

The total cross-section as a function of the centre-of-mass energy for $\Lambda=1 \mathrm{TeV}$ is represented in Fig. 3. It can be seen that, for the scenarios mentioned above, the contribution from the Ztc vertex is about two orders of magnitude smaller than the one from the eetc vertex.

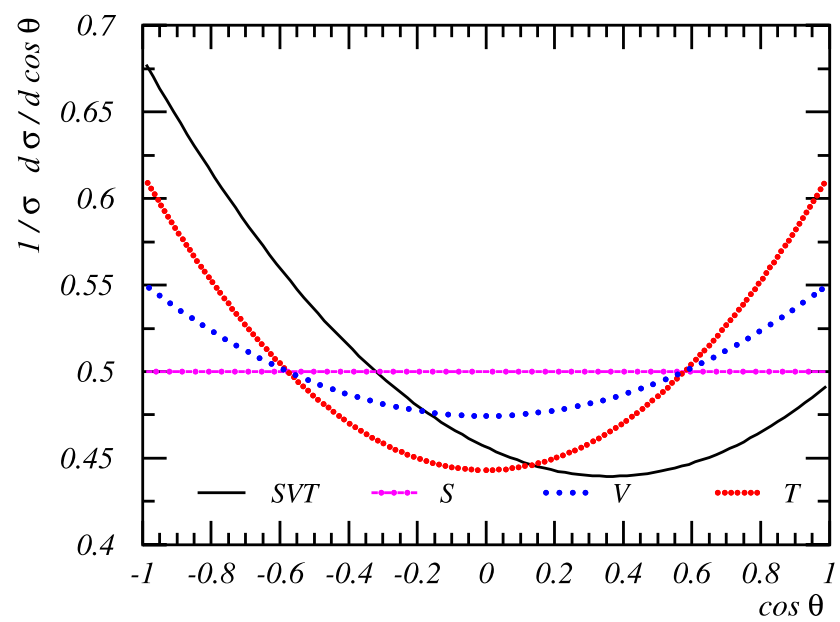

Fig. 2 The differential cross-section $\mathrm{d} \sigma / \mathrm{d} \cos \theta$, normalized to the total cross-section, for the process $\mathrm{e}^{+} \mathrm{e}^{-} \rightarrow \mathrm{t} \overline{\mathrm{c}}$ without ISR, is shown as a function of the cosine of the polar angle of the $\mathrm{t}$ quark, for $m_{\mathrm{t}}=175 \mathrm{GeV} / c^{2}, \Lambda=1 \mathrm{TeV}, \sqrt{s}=206 \mathrm{GeV}$ and the scenarios described in Table 1. The shapes of the differential cross-sections for scenarios $a, V-a$ and $V+a$ are the same as scenario $V$

The Ztc vertex was described within other models by an anomalous coupling, $\kappa_{Z}$, as discussed in Ref. [18]. The couplings $\kappa_{\mathrm{Z}}$ and $a_{j}^{\mathrm{Z}}$ are related by:

$\kappa_{\mathrm{Z}}^{2}=\left[\left(a_{L}^{\mathrm{Z}}\right)^{2}+\left(a_{R}^{\mathrm{Z}}\right)^{2}\right]\left[2 \cos \theta_{\mathrm{W}}\left(\frac{v}{\Lambda}\right)^{2}\right]^{2}$,

where $v=246 \mathrm{GeV}$ is the SM Higgs vacuum expectation value.

The $\mathrm{t}$ quark is expected to decay mainly into $\mathrm{Wb}$. The decays of the $\mathrm{W}$ into both quarks and leptons were considered, giving rise to a hadronic topology $\left(\mathrm{t} \overline{\mathrm{c}} \rightarrow \mathrm{b} \overline{\mathrm{c}} \mathrm{q} \bar{q}^{\prime}\right)$ and a semi-leptonic topology $\left(\mathrm{t} \overline{\mathrm{c}} \rightarrow \mathrm{b} \overline{\mathrm{c}} \ell^{+} \nu_{\ell}\right)$.

\section{Data samples and simulation}

The data were collected with the DELPHI detector during the 1998, 1999 and 2000 LEP2 runs at $\sqrt{s}=189-209 \mathrm{GeV}$ and correspond to a total integrated luminosity of $598.1 \mathrm{pb}^{-1}$.
Table 1 The couplings used in the different scenarios considered in this paper

\begin{tabular}{lllllllrr}
\hline Scenarios & $S_{R R}$ & $V_{R R}$ & $V_{R L}$ & $V_{L R}$ & $V_{L L}$ & $T_{R R}$ & $a_{R}^{Z}$ & $a_{L}^{Z}$ \\
\hline$S V T$ & 1 & 1 & 1 & 1 & 1 & 1 & 0 & 0 \\
$S$ & 1 & 0 & 0 & 0 & 0 & 0 & 0 & 0 \\
$V$ & 0 & 1 & 1 & 1 & 1 & 0 & 0 & 0 \\
$T$ & 0 & 0 & 0 & 0 & 0 & 1 & 0 & 0 \\
$a$ & 0 & 0 & 0 & 0 & 0 & 0 & 1 & 1 \\
$V-a$ & 0 & 1 & 1 & 1 & 1 & 0 & -1 & -1 \\
$V+a$ & 0 & 1 & 1 & 1 & 1 & 0 & 1 & 1 \\
\hline
\end{tabular}


Fig. 3 The total cross-section $\sigma_{\mathrm{tc}}=\sigma\left(\mathrm{e}^{+} \mathrm{e}^{-} \rightarrow \mathrm{t} \overline{\mathrm{c}}+\overline{\mathrm{tc}}\right)$ is shown as a function of the centre-of-mass energy, for $m_{\mathrm{t}}=175 \mathrm{GeV} / c^{2}, \Lambda=1 \mathrm{TeV}$ and for the scenarios described in Table 1. In this scale the cross-sections for scenarios $V-a$ and $V+a$ are indistinguishable from the cross-section for scenario $V$

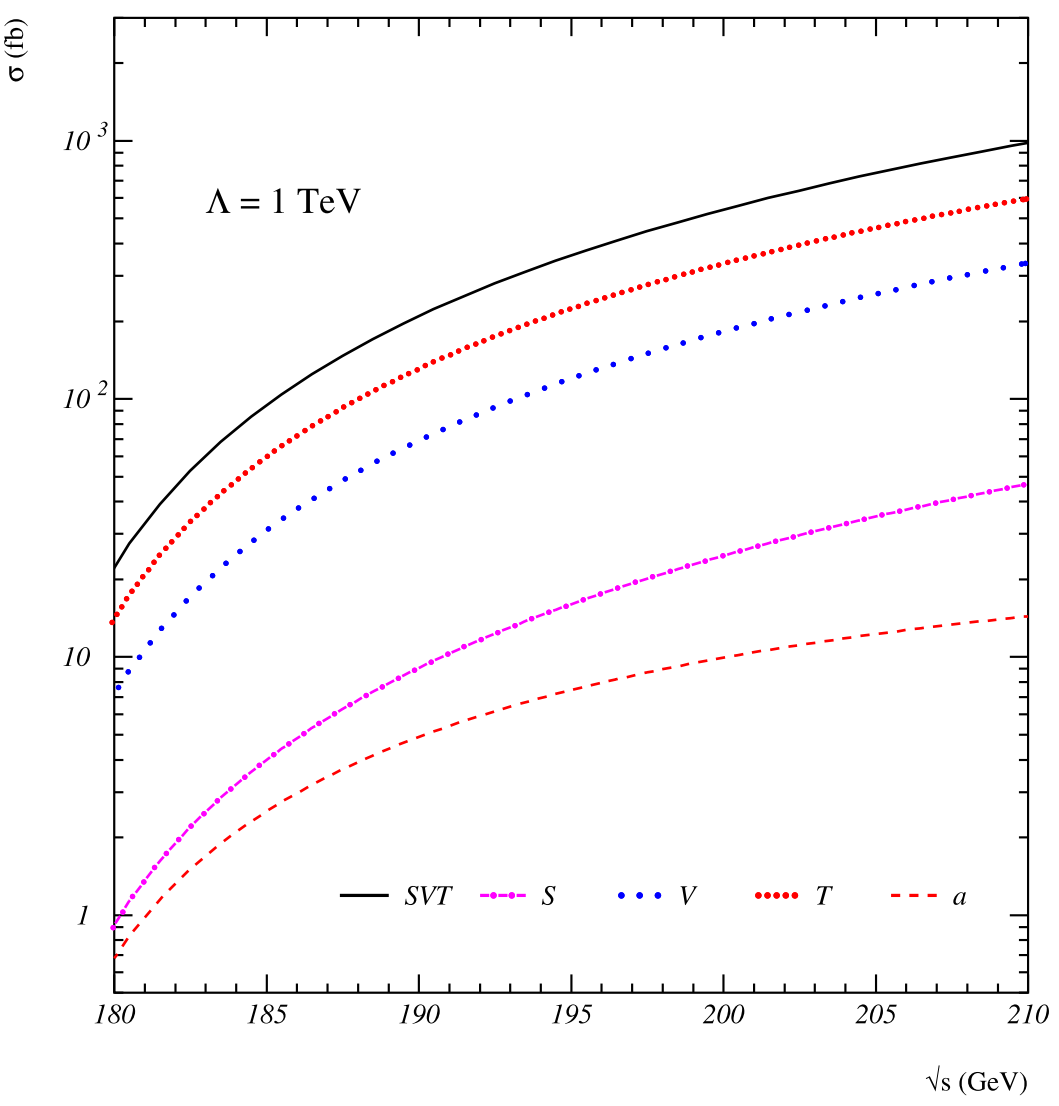

The integrated luminosity collected at each centre-of-mass energy is shown in Table 2.

DELPHI consisted of several sub-detectors in cylindrical layers in the barrel region and was closed by two endcaps that formed the forward region of the detector. The main sub-detectors used for the present analysis were the tracking detectors, the calorimeters and the muon chambers. Starting from the beam pipe, the barrel tracking detectors were a three layer silicon micro-vertex detector (the Vertex Detector), a combined drift/proportional chambers detector (the Inner Detector), the Time Projection Chamber (TPC) which was the main tracking detector and, finally, a 5 layer drift tube detector (the Outer Detector). The forward region was covered by drift chambers (the Forward Chambers A and B). The electromagnetic calorimeters were a sampling calorimeter of lead and gas in the barrel zone, the HighDensity Projection Chamber, and a lead-glass calorimeter with 4532 blocks in each endcap, the Forward Electromagnetic Calorimeter. The Hadron Calorimeter was a sampling iron/gas detector in both the barrel and forward regions, with the iron simultaneously used as the magnet yoke. The Muon Chambers were sets of drift chambers which formed the outer surface of the DELPHI detector and were crucial for identifying muons, essentially the only particles that reached these detectors. A detailed description of the DELPHI detector can be found in Ref. [20, 21]. During the year 2000 data taking, an irreversible failure affected one sector of the TPC, corresponding to $1 / 12$ of its acceptance. The data recorded under these conditions were analysed separately.

The relevant SM background processes were simulated at each centre-of-mass energy using several Monte Carlo generators. All the four-fermion final states (both neutral and charged currents) were generated with WPHACT [22-24], while the particular phase space regions of $\mathrm{e}^{+} \mathrm{e}^{-} \rightarrow \mathrm{e}^{+} \mathrm{e}^{-} \overline{\mathrm{ff}}$
Table 2 Integrated luminosity collected with the DELPHI detector at each centre-of-mass energy. The data collected during the year 2000 with the TPC fully operational were split into two energy bins, below and above $\sqrt{s}=206 \mathrm{GeV}$, with $\langle\sqrt{s}\rangle=204.8 \mathrm{GeV}$ and $\langle\sqrt{s}\rangle=206.6 \mathrm{GeV}$, respectively. The last column, marked by an asterisk, corresponds to data collected with a reduced TPC acceptance

\begin{tabular}{lrrrrrrrr}
\hline Year & 1998 & 1999 & 1999 & 1999 & 1999 & 2000 & 2000 & 2000 \\
\hline$\langle\sqrt{s}\rangle(\mathrm{GeV})$ & 188.6 & 191.6 & 195.5 & 199.5 & 201.6 & 204.8 & 206.6 & $206.3^{*}$ \\
$\mathcal{L}\left(\mathrm{pb}^{-1}\right)$ & 153.0 & 25.1 & 76.0 & 82.7 & 40.2 & 80.0 & 81.9 & 59.2 \\
\hline
\end{tabular}


referred to as $\gamma \gamma$ were generated using PYTHIA 6.1 [25]. The $\mathrm{qq}(\gamma)$ final state was generated with KK2F [26]. Processes giving mainly leptonic final states were also generated, namely Bhabha events with BHWIDE [27], $\mathrm{e}^{+} \mathrm{e}^{-} \rightarrow$ $\mu^{+} \mu^{-}$events with KK2F and $\mathrm{e}^{+} \mathrm{e}^{-} \rightarrow \tau^{+} \tau^{-}$events with KORALZ [28]. The fragmentation and hadronisation of the final-state quarks was handled by PYTHIA 6.1.

For each coupling scenario, signal samples were generated using a modified version of PYTHIA 5.7 [29, 30], where the angular distribution for $\mathrm{t}$ quark production was introduced according to (1) and considering $m_{\mathrm{t}}=175 \mathrm{GeV} / c^{2}$. Samples with t quark masses of $170 \mathrm{GeV}$ and $180 \mathrm{GeV}$ were generated for the evaluation of systematic uncertainties. Initial and final state radiation (ISR and FSR) were taken into account. The final-state quarks fragmentation and hadronisation was handled by JETSET 7.408 [29, 30].

The generated signal and background events were passed through the detailed simulation of the DELPHI detector [21] and then processed with the same reconstruction and analysis programs as the data.

\section{Analysis description}

The analysis consisted of a sequential selection used to identify the event topology and reduce SM background contamination, followed by a probabilistic analysis based on the construction of a discriminant variable. With the exception of a common preselection, the hadronic $\left(\mathrm{t} \overline{\mathrm{c}} \rightarrow \mathrm{b} \overline{\mathrm{c}} \bar{q}^{\prime}\right)$ and the semi-leptonic ( $\mathrm{t} \overline{\mathrm{c}} \rightarrow \mathrm{b} \overline{\mathrm{c}} \ell^{+} v_{\ell}$ ) topologies were considered separately at each step of the analysis.

\subsection{Sequential selection}

A common preselection was adopted for both topologies, followed by specific selection criteria.

Events were preselected requiring at least eight good charged-particles tracks and a visible energy greater than $0.2 \sqrt{s}$, measured at polar angles ${ }^{2}$ above $20^{\circ}$. Good chargedparticles tracks were selected by requiring a momentum above $0.2 \mathrm{GeV} / c$ with a relative error below 1 , and impact parameters along the beam direction and in the transverse plane below $4 \mathrm{~cm} / \sin \theta$ and $4 \mathrm{~cm}$, respectively.

The identification of muons relied on the association of charged particles to signals in the muon chambers and in the hadronic calorimeter and was provided by standard DELPHI algorithms [21], which classified each identified muon as very loose, loose, standard or tight. The identification of

\footnotetext{
${ }^{2}$ In the standard DELPHI coordinate system, the positive $z$ axis is along the electron direction. The polar angle $\theta$ is defined with respect to the $z$ axis. In this paper, polar angle ranges are always assumed to be symmetric with respect to $\theta=90^{\circ}$.
}

electrons and photons was performed by combining information from the electromagnetic calorimeters and the tracking system. Radiation and interaction effects were taken into account by an angular clustering procedure around the main shower [31]. Electron and photon candidates were classified as loose or tight by the identification algorithms.

The search for isolated particles (charged leptons and photons) was done by constructing double cones centered in the direction of charged-particle tracks or neutral energy deposits. The latter ones were defined as calorimetric energy deposits above $0.5 \mathrm{GeV}$, not matched to charged-particles tracks and identified as photon candidates by the standard DELPHI algorithms [21, 31]. For charged leptons (photons), the energy in the region between the two cones, which had half-opening angles of $5^{\circ}$ and $25^{\circ}\left(5^{\circ}\right.$ and $\left.15^{\circ}\right)$, was required to be below $3 \mathrm{GeV}(1 \mathrm{GeV})$, to ensure isolation. All the charged-particle tracks and neutral energy deposits inside the inner cone were associated to the isolated particle. The energy of the isolated particle was then re-evaluated as the sum of the energies inside the inner cone and was required to be above $5 \mathrm{GeV}$. For tight electrons, standard or tight muons or tight photons the above requirements were weakened. In this case only the external cone was used and its angle $\alpha$ was varied according to the energy of the lepton (photon) candidate, down to $2^{\circ}$ for $P_{\ell} \geq 70 \mathrm{GeV} / c$ ( $3^{\circ}$ for $P_{\gamma} \geq 90 \mathrm{GeV} / c$ ), with the allowed energy inside the cone reduced by $\sin \alpha / \sin 25^{\circ}\left(\sin \alpha / \sin 15^{\circ}\right)$.

The topology of each event was defined according to the number of isolated charged leptons identified in the event: events with no isolated charged leptons were classified as hadronic while all the other events were classified as semileptonic. Following the fragmentation and hadronisation, final state quarks were identified as jets. In both topologies, $\mathrm{a} b$ jet identified using the combined $\mathrm{b}$-tag described in Ref. [32], and a low momentum jet from the c quark were expected. The events of the hadronic (semi-leptonic) topology were forced into four (two) jets, ${ }^{3}$ using the Durham jet algorithm [33].

After this common preselection specific selection criteria were applied to both topologies.

\subsubsection{Hadronic topology}

The final state of the hadronic topology $(\mathrm{t} \overline{\mathrm{c}} \rightarrow \mathrm{b} \overline{\mathrm{c}} \overline{\mathrm{q}} \overline{\mathrm{q}})$ is characterized by the presence of four jets, two of them from the $\mathrm{W}$ hadronic decay, and no missing energy. The distributions of relevant variables after the common preselection are shown ${ }^{4}$ in Fig. 4. Due to the high multiplicity expected

\footnotetext{
${ }^{3}$ Isolated charged leptons and isolated photons were excluded in the jet clustering.

${ }^{4}$ For illustration purposes all the simulated signal distributions in Figs. 4-7 and all the plotted distributions in Fig. 8 correspond to scenario $S V T$ (see Table 1).
} 
Fig. 4 Distributions of variables relevant for the sequential selection of the hadronic topology are shown at $\langle\sqrt{s}\rangle=206.6 \mathrm{GeV}$ : (a) ratio between the effective centre-of-mass energy and the centre-of-mass energy; (b) thrust; (c) $-\ln \left(y_{4 \rightarrow 3}\right) ;(\mathbf{d})$ $\chi^{2} / n . d$.f. of the kinematic fit imposing energy-momentum conservation. The WW, q $\overline{\mathrm{q}}(\gamma)$ and "others" labels represent the background contribution from charged-current four-fermion final states generated with WPHACT [22-24], two-fermion final states generated with KK2F [26] and all the other processes mentioned in Sect. 3, respectively. The signal distributions correspond to scenario $S V T$ (see Table 1) and their normalisations are arbitrary, but the same in all plots. The arrows show the applied cuts

DELPHI

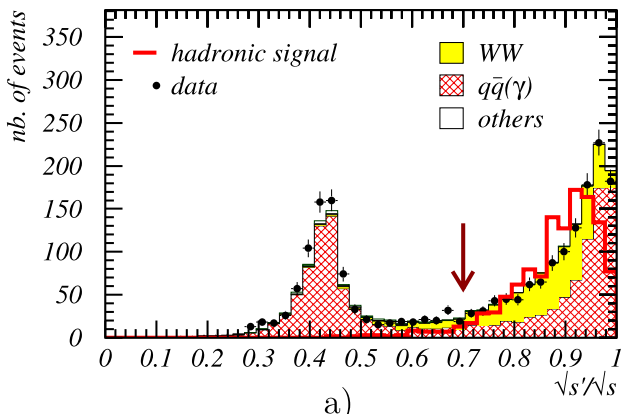

a)

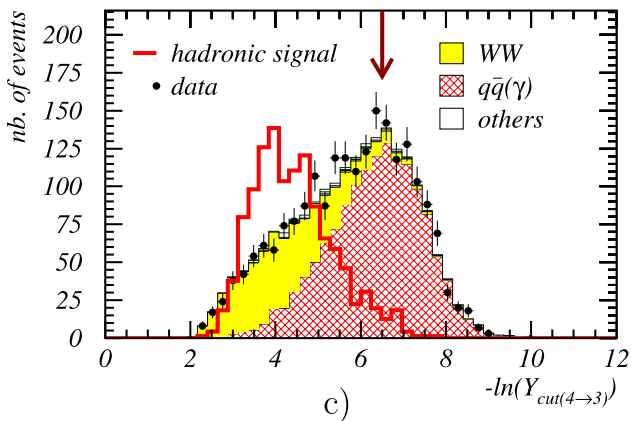

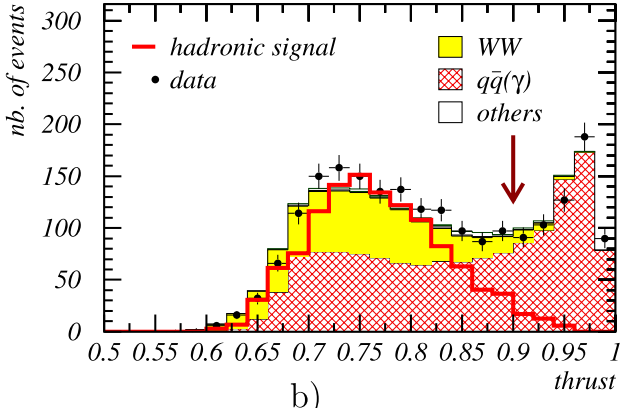

b)

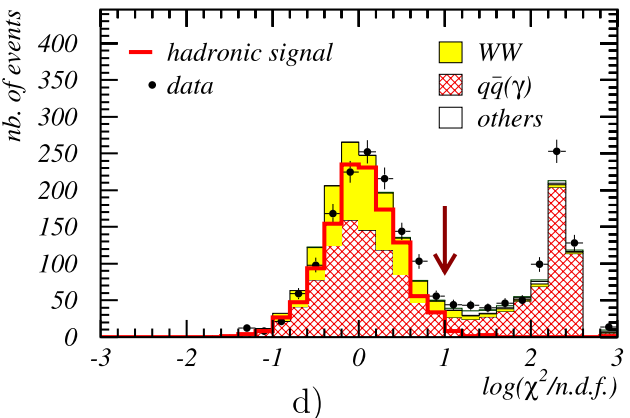

in this topology, the required minimum number of good charged-particles tracks was raised to 25 . The events were required to have an effective centre-of-mass energy [34] $\sqrt{s^{\prime}} \geq 0.7 \sqrt{s}$ and a thrust below 0.9. Events were clustered into four jets and it was required that $-\ln \left(y_{4 \rightarrow 3}\right) \leq 6.5$, where $y_{n \rightarrow n-1}$ is the Durham resolution variable in the transition from $n$ to $n-1$ jets. Assuming a four-jets final state, a kinematic fit requiring energy-momentum conservation was performed. Events with $\chi^{2} / n . d . f$. lower than 10 were accepted.

In Table 3 the number of selected data events, the expected SM background and the signal efficiencies at the end of the sequential selection are shown for the different centreof-mass energies.

\subsubsection{Semi-leptonic topology}

The final state for the semi-leptonic topology ( $\left.\mathrm{t} \overline{\mathrm{c}} \rightarrow \mathrm{b} \overline{\mathrm{c}} \ell^{+} v_{\ell}\right)$ is characterised by the presence of two jets, one isolated and energetic lepton and missing energy. The $b$ jet is expected to be energetic, while the $\mathrm{c}$ jet has low momentum. Events with at least one isolated charged lepton and at least six good charged-particles tracks with TPC information were accepted. The particles of the events, excluding the isolated leptons, were clustered into two jets using the Durham algorithm and the events were divided into three mutually exclusive samples according to the identified flavour of the most energetic lepton: events with a tight electron ("e sample"), events with a standard or tight muon (" $\mu$ sample") and events in which no unambiguous lepton was present ("no-id sample"). ${ }^{5}$

In the e and no-id samples, photons converting in the tracking system were vetoed by requiring that the lepton had left a signal in at least two layers of the vertex detector. Contamination from Bhabha and $\gamma \gamma$ events was reduced in the e sample by imposing that the lepton was above $25^{\circ}$ in polar angle and that the isolation angle, defined as the angle between the lepton and the nearest jet, isolated photon or other isolated charged leptons, was greater than $10^{\circ}$. For the no-id sample, the contribution from these backgrounds was reduced by keeping only events with exactly one isolated lepton with a polar angle greater than $25^{\circ}$, momentum above $0.075 \sqrt{s}$ and an isolation angle of at least $20^{\circ}$. The distributions of relevant variables after these cuts are shown in Fig. 5. The $q \bar{q}$ background contamination, in the e and no-id samples, was further reduced by requiring a missing momentum above $10 \mathrm{GeV} / c$ pointing above $25^{\circ}$ in polar angle.

Assuming a jj $\ell v$ final state and assigning the missing momentum to the undetected neutrino, a kinematic fit requiring energy-momentum conservation was applied in all three samples. Events with $\chi^{2} / n . d . f$. lower than 10 were accepted.

\footnotetext{
${ }^{5}$ Notice that, according to these definitions, the e and $\mu$ samples also contain the tauonic events in which the $\tau$ decayed, respectively, into a e (if classified as tight) or a $\mu$ (if classified as standard or tight).
} 
Table 3 Number of selected data events, expected SM background and signal efficiencies, $\varepsilon$, (in percent and convoluted with the branching ratio of the $\mathrm{W}$ boson) after the sequential selection. The expected background numbers are shown with their statistical errors. The effi- ciencies have been computed for the different coupling scenarios according to Table 1 and the extreme values are shown. The statistical errors on the efficiency are smaller than $1.3 \%$ and $0.6 \%$ for the hadronic and semi-leptonic topologies, respectively

\begin{tabular}{|c|c|c|c|c|c|c|c|c|}
\hline$\langle\sqrt{s}\rangle(\mathrm{GeV})$ & 188.6 & 191.6 & 195.5 & 199.5 & 201.6 & 204.8 & 206.6 & $206.3^{*}$ \\
\hline \multicolumn{9}{|c|}{ Hadronic topology } \\
\hline data & 1165 & 211 & 613 & 637 & 306 & 599 & 606 & 400 \\
\hline \multirow[t]{2}{*}{ background } & 1216.1 & 197.0 & 589.5 & 637.7 & 299.6 & 610.6 & 612.7 & 444.1 \\
\hline & \pm 14.4 & \pm 2.3 & \pm 6.6 & \pm 7.0 & \pm 3.3 & \pm 6.6 & \pm 6.5 & \pm 4.8 \\
\hline$\varepsilon \min .(\%)$ & 46.5 & 42.8 & 42.8 & 50.9 & 50.9 & 51.5 & 51.5 & 50.5 \\
\hline$\varepsilon \max .(\%)$ & 48.2 & 48.9 & 48.9 & 54.0 & 54.0 & 55.6 & 55.6 & 54.5 \\
\hline \multicolumn{9}{|c|}{ Semi-leptonic topology-e sample } \\
\hline data & 259 & 37 & 140 & 151 & 80 & 166 & 137 & 106 \\
\hline \multirow[t]{2}{*}{ background } & 290.8 & 46.0 & 142.8 & 157.1 & 75.9 & 155.2 & 158.2 & 109.6 \\
\hline & \pm 5.2 & \pm 0.8 & \pm 2.5 & \pm 2.8 & \pm 1.3 & \pm 2.7 & \pm 2.8 & \pm 2.0 \\
\hline$\varepsilon \min .(\%)$ & 6.5 & 6.1 & 6.1 & 6.4 & 6.4 & 6.5 & 6.5 & 6.2 \\
\hline$\varepsilon \max .(\%)$ & 7.6 & 7.3 & 7.3 & 7.2 & 7.2 & 7.1 & 7.6 & 7.1 \\
\hline \multicolumn{9}{|c|}{ Semi-leptonic topology $-\mu$ sample } \\
\hline data & 423 & 75 & 226 & 259 & 111 & 240 & 220 & 169 \\
\hline \multirow[t]{2}{*}{ background } & 432.9 & 75.4 & 225.6 & 246.7 & 118.4 & 232.8 & 244.3 & 169.9 \\
\hline & \pm 6.5 & \pm 1.1 & \pm 3.3 & \pm 3.6 & \pm 1.7 & \pm 3.3 & \pm 3.5 & \pm 2.5 \\
\hline$\varepsilon \min .(\%)$ & 10.6 & 10.5 & 10.5 & 10.3 & 10.3 & 10.7 & 10.5 & 9.9 \\
\hline$\varepsilon \max .(\%)$ & 11.5 & 11.6 & 11.6 & 11.4 & 11.4 & 11.1 & 11.5 & 10.8 \\
\hline \multicolumn{9}{|c|}{ Semi-leptonic topology—no-id sample } \\
\hline data & 308 & 49 & 140 & 135 & 67 & 145 & 148 & 92 \\
\hline \multirow[t]{2}{*}{ background } & 286.2 & 45.4 & 133.9 & 146.8 & 72.0 & 141.1 & 141.7 & 104.5 \\
\hline & \pm 7.5 & \pm 1.2 & \pm 3.3 & \pm 3.6 & \pm 1.7 & \pm 3.3 & \pm 3.4 & \pm 2.5 \\
\hline$\varepsilon \min .(\%)$ & 2.7 & 2.6 & 2.6 & 2.8 & 2.8 & 2.9 & 3.3 & 2.7 \\
\hline$\varepsilon \max .(\%)$ & 3.5 & 3.4 & 3.4 & 3.3 & 3.3 & 3.3 & 3.6 & 3.4 \\
\hline \multicolumn{9}{|l|}{ Total } \\
\hline data & 2155 & 372 & 1119 & 1182 & 564 & 1150 & 1111 & 767 \\
\hline \multirow[t]{2}{*}{ background } & 2226.0 & 363.8 & 1091.8 & 1188.3 & 565.9 & 1139.7 & 1156.9 & 828.1 \\
\hline & \pm 18.2 & \pm 2.9 & \pm 8.5 & \pm 9.1 & \pm 4.3 & \pm 8.5 & \pm 8.6 & \pm 6.3 \\
\hline$\varepsilon \min .(\%)$ & 67.5 & 62.3 & 62.3 & 71.3 & 71.3 & 72.7 & 72.6 & 69.8 \\
\hline$\varepsilon \max .(\%)$ & 69.3 & 69.8 & 69.8 & 75.0 & 75.0 & 76.7 & 77.5 & 73.6 \\
\hline
\end{tabular}

In Table 3 the number of selected data events, the expected SM background and the signal efficiencies are shown at the end of the sequential selection.

\subsection{Discriminant selection}

After the sequential analysis, the main background consisted of $\mathrm{W}^{+} \mathrm{W}^{-}$events, which are similar to the signal and have the same final state topology. A separation is possible, based on the different kinematic properties and on jet-flavour tag- ging techniques. Furthermore, the $\mathrm{W}$ and t quark mass constraints can be used to separate signal and background.

\subsubsection{Hadronic topology}

In order to identify the $\mathrm{b}$ and $\mathrm{c}$ jets and determine the kinematic properties of the $\mathrm{t}$ quark and of the $\mathrm{W}$ boson, several possible jet assignment schemes were studied:

1. the most energetic jet is assigned to the $b$ quark and the least energetic one to the c quark; 
Fig. 5 Distributions of variables relevant for the sequential selection of the semi-leptonic topology after the common preselection are shown at $\langle\sqrt{s}\rangle=206.6 \mathrm{GeV}$. e sample: (a) missing momentum; (b) polar angle of the missing momentum (after applying the cut on the missing momentum distribution); $\mu$ sample:

(c) lepton polar angle; (d) lepton isolation angle; no-id sample: (e) missing momentum; (f) polar angle of the missing momentum (after applying the cut on the missing momentum distribution). The WW, $\mathrm{q} \overline{\mathrm{q}}(\gamma)$ and "others" labels have the same meaning as in Fig. 4. The signal distributions correspond to scenario $S V T$ (see Table 1) and their normalisations are arbitrary, but the same in all plots. The arrows show the applied cuts

Table 4 Fraction of the correct assignments of jets to quarks for simulated signal events of the hadronic topology at $\sqrt{s}=189 \mathrm{GeV}$ and $\sqrt{s}=206 \mathrm{GeV}$, using the four jet assignment schemes explained in the text
DELPHI
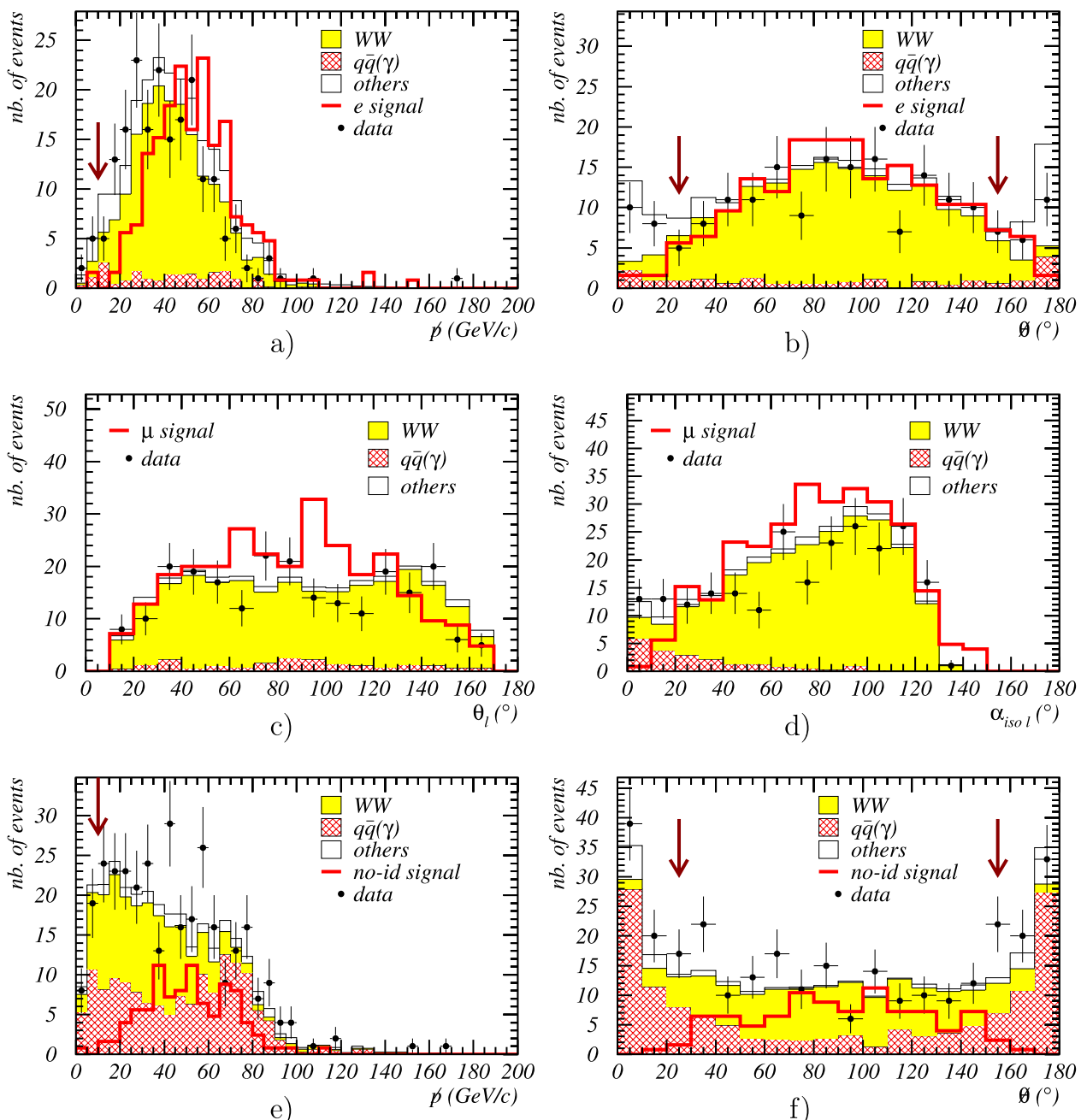

\begin{tabular}{lllll}
\hline Scheme & 1 & 2 & 3 & 4 \\
\hline$\sqrt{s}=189 \mathrm{GeV}$ & & & & \\
$\varepsilon_{b}(\%)$ & 52.4 & 52.4 & 72.5 & 72.5 \\
$\varepsilon_{c}(\%)$ & 45.5 & 43.4 & 41.9 & 40.7 \\
$\sqrt{s}=206 \mathrm{GeV}$ & & & & \\
$\varepsilon_{b}(\%)$ & 53.3 & 53.3 & 68.0 & 68.0 \\
$\varepsilon_{c}(\%)$ & 51.2 & 51.0 & 47.1 & 44.8
\end{tabular}

2. the most energetic jet is assigned to the $b$ quark and the jets that minimise $\left|m_{\mathrm{jj}}-m_{\mathrm{W}}\right|$, where $m_{\mathrm{jj}}$ is the invariant mass of two of the three remaining jets and $m_{\mathrm{W}}=$ $80.4 \mathrm{GeV} / c^{2}$, are assigned to the $\mathrm{W}$ boson;

3. the jet with the highest $b$-tag value is assigned to the $b$ quark and the least energetic one of the remaining three to the c quark;
4. the jet with the highest $b$-tag value is assigned to the $b$ quark and, from the three remaining, the jets that minimise $\left|m_{\mathrm{jj}}-m_{\mathrm{W}}\right|$ are assigned to the $\mathrm{W}$ boson.

The correct assignment of jets to quarks was studied with simulated signal samples at $\sqrt{s}=189 \mathrm{GeV}$ and $\sqrt{s}=$ $206 \mathrm{GeV}$. Correct assignment was defined based on the angle $\alpha_{\mathrm{qj}}$ between the quark and jet direction, requiring 
Fig. 6 Distributions of variables relevant for the hadronic topology after the sequential selection at $\langle\sqrt{s}\rangle=206.6 \mathrm{GeV}$ : (a) b-tag of the event; (b) b jet momentum; (c) reconstructed $\mathrm{W}$ boson mass; (d) reconstructed t quark mass. The (a), (b) and (c) distributions were used as PDF to construct the discriminant variable for the hadronic topology. The signal distributions correspond to scenario $S V T$ (see Table 1) and their normalisations are arbitrary, but the same in all plots
DELPHI
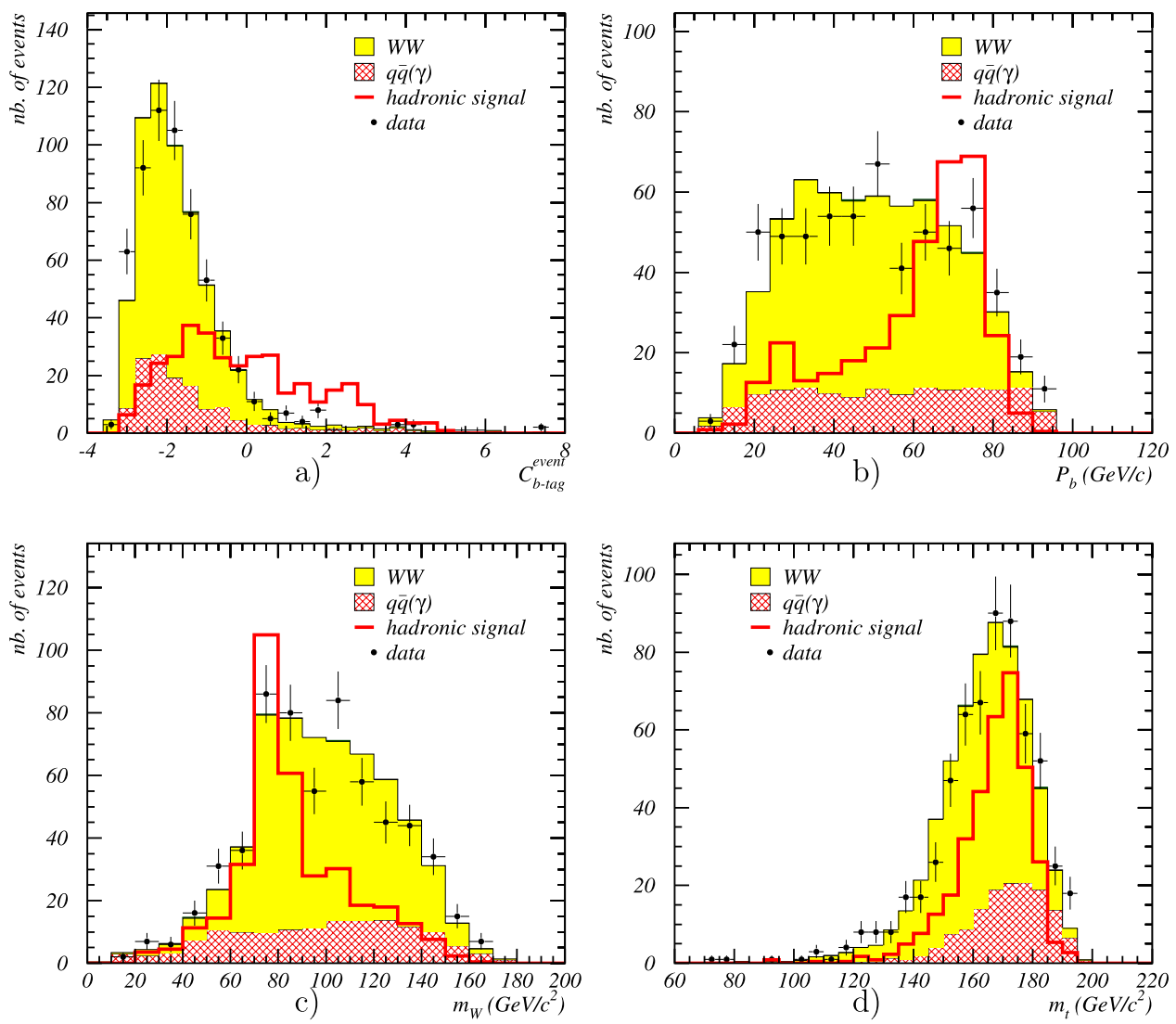

$\cos \alpha_{\mathrm{qj}} \geq 0$.9. The results are presented in Table 4: higher efficiencies for the $\mathrm{b}$ quark assignment are obtained with schemes 3 and 4 . Scheme 3 was adopted since it also yields the best discrimination between signal and background.

Signal and background-like probabilities were assigned to each event based on Probability Density Functions (PDF) constructed with the following variables:

- the event b-tag value, $C_{\mathrm{b}-\text { tag }}^{\text {event}}$;

- the b momentum, $P_{\mathrm{b}}$;

- the $\mathrm{W}$ reconstructed mass, $m_{\mathrm{W}}$.

The distributions of these variables are shown in Fig. 6 for data, expected background and signal at $\langle\sqrt{s}\rangle=206.6 \mathrm{GeV}$. For each of these variables, the corresponding PDF for the signal $\left(P_{S}^{i}\right)$ and background $\left(P_{B}^{i}\right)$ were estimated. For each event, a signal likelihood $\left(P_{S}=\prod_{i} P_{S}^{i}\right)$ and background likelihood $\left(P_{B}=\prod_{i} P_{B}^{i}\right)$ were computed assuming no correlations. The discriminant variable was defined as $\ln \mathcal{L}_{R}=$ $\ln \left(P_{S} / P_{B}\right)$.

\subsubsection{Semi-leptonic topology}

A discriminant variable was constructed using signal $\left(P_{S}^{i}\right)$ and background $\left(P_{B}^{i}\right)$ PDFs estimated from the following variables:
- the angle between the two jets, $\alpha_{j_{1} j_{2}}$;

- the angle between the lepton and the neutrino, $\alpha_{\ell_{\nu}}$;

- the reconstructed mass of the two jets, $m_{j_{1} j_{2}}$;

- the reconstructed mass of the jet with the highest b-tag, the lepton and the neutrino, $m_{\mathrm{b} \ell \nu}$;

- the reconstructed W mass, $m_{\ell v}$;

- the ratio of the jet momenta, $P_{j_{2}} / P_{j_{1}}$;

- the b-tag of the most energetic jet, $C_{\mathrm{b} \text {-tag }}^{j_{1}}$;

- the product of the lepton charge and the cosine of the lepton polar angle, $Q_{\ell} \times \cos \theta_{\ell}$;

- $-\ln y_{2 \rightarrow 1}$, where $y_{2 \rightarrow 1}$ is the Durham resolution variable in the transition from two to one jet.

Distributions of some of these variables are shown in Fig. 7 for $\langle\sqrt{s}\rangle=206.6 \mathrm{GeV}$.

\section{Results}

The discriminant variables obtained in the different search channels are shown in Fig. 8, for $\langle\sqrt{s}\rangle=206.6 \mathrm{GeV}$. As no signal was found in any of the analysis channels, limits at $95 \%$ confidence level (CL) on the energy scale $\Lambda$ were derived for each of the scenarios in Table 1. The limits were obtained using the modified frequentist likelihood 
Fig. 7 Distributions of variables relevant for the semi-leptonic topology after the sequential selection at $\langle\sqrt{s}\rangle=206.6 \mathrm{GeV}$. In the left column: angle between the lepton and the neutrino; in the right column: $b$-tag of most energetic jet; (a, b) e sample; (c, d) $\mu$ sample; (e, f) no-id sample. The signal distributions correspond to scenario $S V T$ (see Table 1) and their normalisations are arbitrary, but the same in all plots
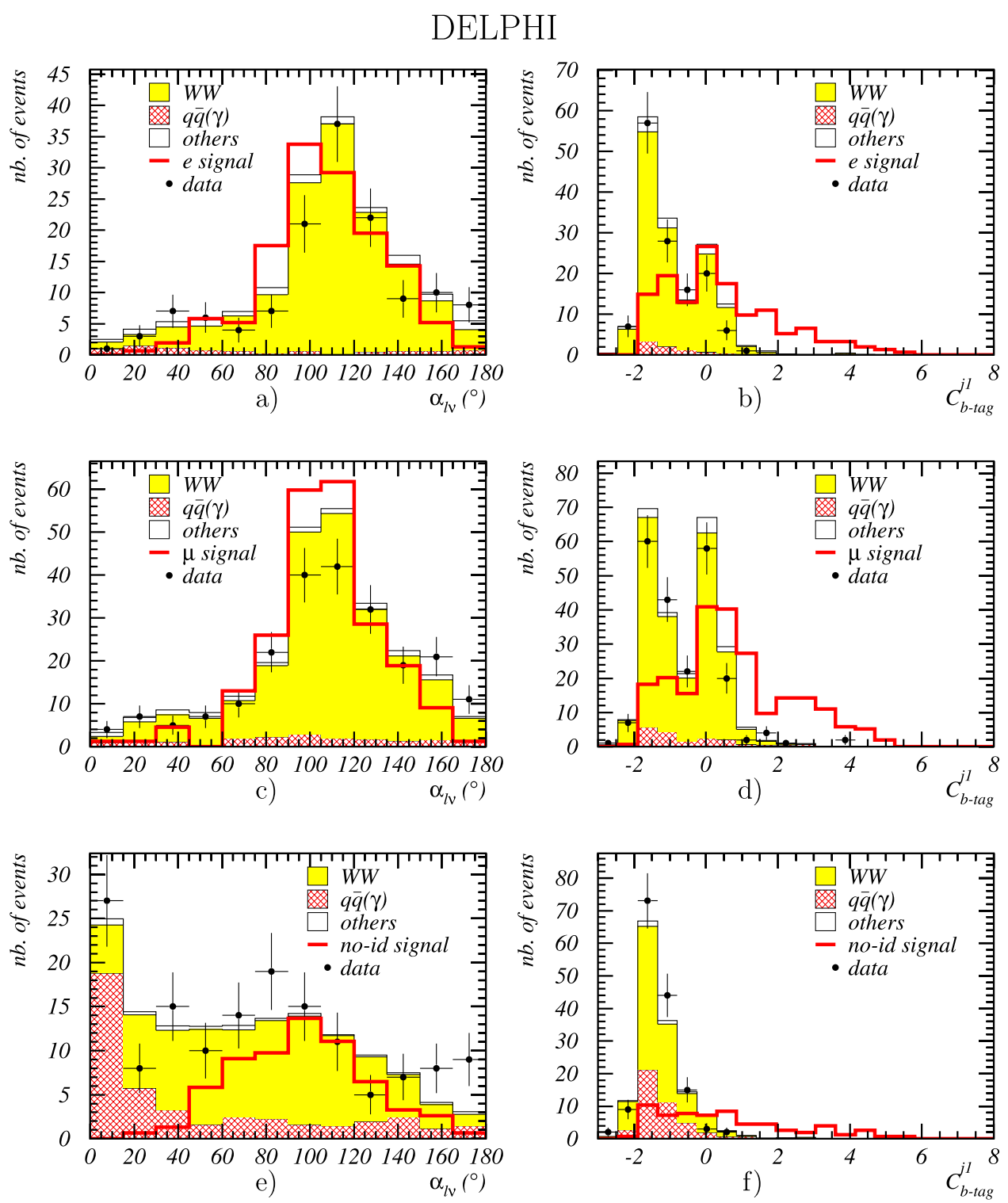

ratio method [35], taking into account the observed and expected event counts, the signal efficiencies and the shapes of the discriminant variables in data, background and signal. The expected limit was computed as the median limit for experiments without signal contributions. The $\pm 1 \sigma$ values around the expected median limit were also computed. In order to avoid non-physical fluctuations in the distributions of the discriminant variables, due to the limited statistics of the generated events, a smoothing procedure was adopted. The limits were evaluated assuming $m_{\mathrm{t}}=175 \mathrm{GeV} / c^{2}$, which allows direct comparison with other published results. The results, obtained with the contribution of all the systematic uncertainties described in the next paragraph, are presented in Table 5. The observed and expected limits are statistically compatible and the maximum difference is about $1 \sigma$.
The effect of a change in the $t$ quark mass was studied at two extreme energies (188.6 and $204.8 \mathrm{GeV}$ ) and two extreme scenarios $(S V T$ and $a$ ), considering the values 170 and $180 \mathrm{GeV} / c^{2}$ for $m_{\mathrm{t}}$. The estimated relative changes in the limits were about $+10 \%$ for $m_{\mathrm{t}}=170 \mathrm{GeV} / c^{2}$ and $-14 \%$ for $m_{\mathrm{t}}=180 \mathrm{GeV} / c^{2}$. For scenarios $S$ and $T$, in which only one coupling is non-zero, limits at $95 \% \mathrm{CL}$ on the ratio between the coupling and $\Lambda^{2}$ can be directly obtained from (2):

$$
\begin{aligned}
& \left(\frac{T}{\Lambda^{2}}\right)_{\mathrm{obs}} \leq 6.90 \times 10^{-7} \mathrm{GeV}^{-2}, \\
& \left(\frac{T}{\Lambda^{2}}\right)_{\exp } \leq 6.83 \times 10^{-7} \mathrm{GeV}^{-2},
\end{aligned}
$$


Fig. 8 Distributions of the discriminant variable $\ln \mathcal{L}_{R}$ for data, expected background and signal after the sequential selection at $\langle\sqrt{s}\rangle=206.6 \mathrm{GeV}$ : (a) hadronic topology; semi-leptonic topology: (b) e sample; (c) $\mu$ sample; (d) no-id sample. These distributions correspond to scenario $S V T$ (see Table 1). The signal normalisation is arbitrary, but the same in all plots
DELPHI
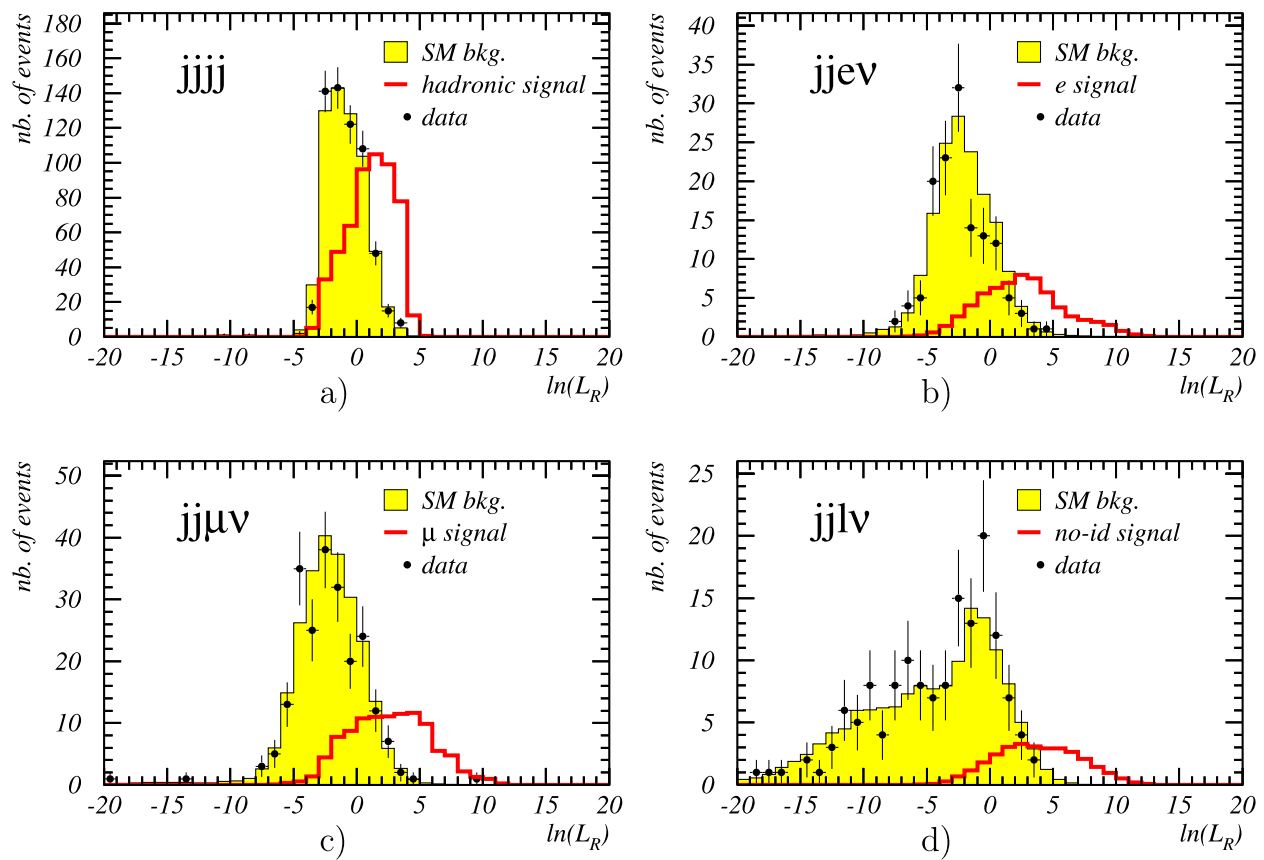

Table 5 Observed and expected $95 \% \mathrm{CL}$ lower limits on $\Lambda(\mathrm{GeV})$ for the different scenarios, assuming $m_{\mathrm{t}}=175 \mathrm{GeV} / c^{2}$. The $\pm 1 \sigma$ values around the expected median limit are also shown

\begin{tabular}{|c|c|c|c|c|c|c|c|c|c|c|c|c|}
\hline \multirow[t]{2}{*}{ Scenario } & \multicolumn{4}{|c|}{ Hadronic topology } & \multicolumn{4}{|c|}{ Semi-leptonic topology } & \multicolumn{4}{|c|}{ Combined topologies } \\
\hline & obs. & $-1 \sigma$ & exp. & $+1 \sigma$ & obs. & $-1 \sigma$ & exp. & $+1 \sigma$ & obs. & $-1 \sigma$ & exp. & $+1 \sigma$ \\
\hline$S V T$ & 1218 & 1268 & 1180 & 1097 & 1315 & 1406 & 1301 & 1203 & 1402 & 1468 & 1366 & 1264 \\
\hline$S$ & 577 & 604 & 556 & 520 & 647 & 647 & 603 & 555 & 685 & 693 & 641 & 593 \\
\hline$V$ & 953 & 1003 & 933 & 863 & 997 & 1069 & 997 & 921 & 1073 & 1141 & 1068 & 980 \\
\hline$T$ & 1069 & 1117 & 1045 & 969 & 1124 & 1232 & 1142 & 1052 & 1204 & 1300 & 1210 & 1114 \\
\hline$a$ & 436 & 462 & 430 & 400 & 472 & 513 & 475 & 436 & 499 & 535 & 499 & 459 \\
\hline$V-a$ & 961 & 1009 & 941 & 877 & 1018 & 1093 & 1018 & 938 & 1093 & 1163 & 1083 & 998 \\
\hline$V+a$ & 935 & 983 & 915 & 851 & 988 & 1078 & 1002 & 921 & 1058 & 1130 & 1058 & 974 \\
\hline
\end{tabular}

$\left(\frac{S}{\Lambda^{2}}\right)_{\mathrm{obs}} \leq 2.13 \times 10^{-6} \mathrm{GeV}^{-2}$,

$\left(\frac{S}{\Lambda^{2}}\right)_{\exp } \leq 2.43 \times 10^{-6} \mathrm{GeV}^{-2}$

The limit obtained in scenario $a$, involving only the $a_{j}^{Z}$ couplings, can be converted into a limit on the anomalous coupling $\kappa_{\mathrm{Z}}$ (see (3)), ${ }^{6}$ yielding $\kappa_{\mathrm{Z}}^{\text {obs }} \leq 0.43$.

The evaluation of the limits was performed taking into account systematic uncertainties, which affect the background estimation and the signal efficiency. The stability

\footnotetext{
${ }^{6}$ Notice that in Ref. [13-17] the contribution from the processes $\mathrm{e}^{+} \mathrm{e}^{-} \rightarrow \mathrm{t} \overline{\mathrm{u}}$ and $\mathrm{e}^{+} \mathrm{e}^{-} \rightarrow \overline{\mathrm{t} u}$ are also considered. This was taken into account in the limits conversion.
}

of the sequential analysis was studied by changing the cut values in the most relevant variables by typically $10 \%$. The maximum relative change in the limit was about $2 \%$. Different parameterisations inside PYTHIA were used to study the dependence of the efficiency on the hadronisation and fragmentation of the jets associated to heavy quarks. The Lund symmetric fragmentation, the Bowler space-time picture of string evolution and the Peterson/SLAC function were considered. ${ }^{7}$ The maximum relative change in the limit was about $2 \%$. The effect of PDF binning and smoothing procedures was studied and the maximum relative change in the limit was about $3 \%$. A similar study was performed for the discriminant variables and the maximum relative change in the limit was about $6 \%$.

\footnotetext{
${ }^{7}$ See $[29,30]$ for more details
} 


\section{Conclusions}

Single top quark production via contact interactions was searched for using data collected by the DELPHI detector at centre-of-mass energies ranging from $189 \mathrm{GeV}$ to $209 \mathrm{GeV}$, corresponding to an integrated luminosity of $598.1 \mathrm{pb}^{-1}$. The coupling scenarios listed in Table 1 were considered and a dedicated analysis was developed. No evidence for a signal was found. Limits at $95 \%$ confidence level on the new physics energy scale $\Lambda$ were obtained and the observed values for different scenarios range from $499 \mathrm{GeV}$ to $1402 \mathrm{GeV}$ (see Table 5). The observed limit on the anomalous coupling $\kappa_{\mathrm{Z}}$, obtained from the conversion of scenario $a$ limit, is $\kappa_{\mathrm{Z}}^{\mathrm{obs}} \leq 0.43$.

The L3 collaboration also searched for single t quark production via contact interactions and the results [16] are similar to those presented here. The converted limit on the anomalous coupling $\kappa_{\mathrm{Z}}$ agrees with those presented by the four LEP collaborations [13-17] in the framework of Ref. [18].

Acknowledgements We are greatly indebted to our technical collaborators, to the members of the CERN-SL Division for the excellent performance of the LEP collider, and to the funding agencies for their support in building and operating the DELPHI detector.

We acknowledge in particular the support of

Austrian Federal Ministry of Education, Science and Culture, GZ 616.364/2-III/2a/98,

FNRS-FWO, Flanders Institute to encourage scientific and technological research in the industry (IWT) and Belgian Federal Office for Scientific, Technical and Cultural affairs (OSTC), Belgium,

FINEP, CNPq, CAPES, FUJB and FAPERJ, Brazil,

Ministry of Education of the Czech Republic, project LC527,

Academy of Sciences of the Czech Republic, project AV0Z10100502,

Commission of the European Communities (DG XII),

Direction des Sciences de la Matière, CEA, France,

Bundesministerium für Bildung, Wissenschaft, Forschung und Technologie, Germany,

General Secretariat for Research and Technology, Greece,

National Science Foundation (NWO) and Foundation for Research on

Matter (FOM), The Netherlands,

Norwegian Research Council,

State Committee for Scientific Research, Poland, SPUB-M/CERN/PO3/ DZ296/2000, SPUB-M/CERN/PO3/DZ297/2000, 2P03B 10419 and 2P03B 69 23(2002-2004),

FCT-Fundação para a Ciência e Tecnologia, Portugal,

Vedecka grantova agentura MS SR, Slovakia, Nr. 95/5195/134,

Ministry of Science and Technology of the Republic of Slovenia,

CICYT, Spain, AEN99-0950 and AEN99-0761,

The Swedish Research Council,

The Science and Technology Facilities Council, UK,

Department of Energy, USA, DE-FG02-01ER41155,

EEC RTN contract HPRN-CT-00292-2002.

Open Access This article is distributed under the terms of the Creative Commons Attribution Noncommercial License which permits any noncommercial use, distribution, and reproduction in any medium, provided the original author(s) and source are credited.

\section{References}

1. The Tevatron Electroweak Working Group for the CDF and DØ Collaborations, Combination of $\mathrm{CDF}$ and $\mathrm{D} \emptyset$ results on the mass of the top quark using up to $5.6 \mathrm{fb}^{-1}$ of data. arXiv:1007.3178, July 2010

2. E. Boos et al., Phys. Lett. B 326, 190 (1994)

3. S.L. Glashow, J. Iliopoulos, L. Maiani, Phys. Rev. D 2, 1285 (1970)

4. C.S. Huang, X.H. Wu, S.H. Zhu, Phys. Lett. B 452, 143 (1999)

5. J.A. Aguilar-Saavedra, Phys. Rev. D 67, 035003 (2003). Erratumibid. Phys. Rev. D 69, 099901 (2004)

6. D. Atwood, L. Reina, A. Soni, Phys. Rev. D 53, 1199 (1996)

7. D. Atwood, L. Reina, A. Soni, Phys. Rev. D 55, 3156 (1997)

8. S. Bejar, J. Guasch, J. Sola, Nucl. Phys. B 600, 21 (2001)

9. J.J. Liu, C.S. Li, L.L. Yang, L.G. Jin, Phys. Lett. B 599, 92 (2004)

10. D. Delepine, S. Khalil, Phys. Lett. B 599, 62 (2004)

11. J. Guasch, J. Sola, Nucl. Phys. B 562, 3 (1999)

12. J.M. Yang, B.L. Young, X. Zhang, Phys. Rev. D 58, 055001 (1998)

13. R. Barate et al. (ALEPH Collaboration), Phys. Lett. B 494, 33 (2000)

14. A. Heister et al. (ALEPH Collaboration), Phys. Lett. B 543, 173 (2002)

15. J. Abdallah et al. (DELPHI Collaboration), Phys. Lett. B 590, 21 (2004)

16. P. Achard et al. (L3 Collaboration), Phys. Lett. B 549, 290 (2002)

17. G. Abbiendi et al. (OPAL Collaboration), Phys. Lett. B 521, 181 (2001)

18. V.F. Obraztsov, S.R. Slabospitsky, O.P. Yushchenko, Phys. Lett. B 426, 393 (1998)

19. S. Bar-Shalom, J. Wudka, Phys. Rev. D 60, 094016 (1999)

20. P. Aarnio et al. (DELPHI Collaboration), Nucl. Instrum. Methods A 303, 233 (1991)

21. P. Abreu et al. (DELPHI Collaboration), Nucl. Instrum. Methods A 378, 57 (1996)

22. E. Accomando, A. Ballestrero, Comput. Phys. Commun. 99, 270 (1997)

23. E. Accomando, A. Ballestrero, E. Maina, Comput. Phys. Commun. 150, 166 (2003)

24. A. Ballestrero, R. Chierici, F. Cossutti, E. Migliore, Comput. Phys. Commun. 152, 175 (2003)

25. T. Sjöstrand et al., Comput. Phys. Commun. 135, 238 (2001)

26. S. Jadach, B.F.L. Ward, Z. Was, Comput. Phys. Commun. 130, $260(2000)$

27. S. Jadach, W. Płaczek, B.F.L. Ward, Phys. Lett. B 390, 298 (1997)

28. S. Jadach, B.F.L. Ward, Z. Was, Comput. Phys. Commun. 79, 503 (1994)

29. T. Sjöstrand, Comput. Phys. Commun. 82, 74 (1994)

30. T. Sjöstrand, PYTHIA 5.7 and JETSET 7.4, CERN-TH/7112-93

31. F. Cossutti et al., REMCLU: a package for the Reconstruction of ElectroMagnetic CLUsters at LEP200, DELPHI Note 2000-164 PROG 242 (available at http://delphiwww.cern.ch/ pubxx/delnote/public/2000_164_prog_242.ps.gz)

32. J. Abdallah et al. (DELPHI Collaboration), Eur. Phys. J. C 32, 185 (2004)

33. S. Catani et al., Phys. Lett. B 269, 432 (1991)

34. P. Abreu et al., Nucl. Instrum. Methods A 427, 487 (1999)

35. A.L. Read, CERN report 2000-005, 81, 2000 